\title{
Overexpression of stromal interaction molecule 1 may promote epithelial-mesenchymal transition and indicate poor prognosis in gastric cancer
}

\author{
GUOBIN WU, YONG LI, BIBO TAN, LIQIAO FAN, QUN ZHAO, YÜ LIU and ZHIDONG ZHANG \\ Department of General Surgery, The Fourth Affiliated Hospital, \\ Hebei Medical University, Shijiazhuang, Hebei 050011, P.R. China
}

Received May 2, 2016; Accepted March 23, 2017

DOI: $10.3892 / \mathrm{mmr} .2017 .6607$

\begin{abstract}
The aim of the present study was to investigate the prognostic significance of stromal interaction molecule 1 (STIM1) expression in gastric cancer (GC) and examine the association between STIM1 and epithelial-mesenchymal transition (EMT). Immunohistochemical staining was performed to detect STIM1, E-cadherin, $\beta$-catenin and matrix metalloproteinase-9 (MMP-9) in 170 GC and 35 adjacent healthy gastric tissue samples. Positive staining of STIM1, E-cadherin, $\beta$-catenin and MMP-9 in GC tissues was significantly greater compared with adjacent healthy tissues $(\mathrm{P}<0.05)$. Clinicopathological analysis revealed that STIM1 expression was significantly associated with LNM $(\mathrm{P}<0.001)$ and tumor-node-metastasis stage $(\mathrm{P}=0.01)$. The overall survival rate was significantly reduced in STIM1-positive compared with STIM1-negative patients $(\mathrm{P}=0.043)$. Cox regression analysis indicated that STIM1 expression and LNM were independent prognostic factors for GC. Chi-square tests suggested that STIM1 expression in GC tissues was significantly associated with E-cadherin $(\mathrm{P}<0.001)$ and $\beta$-catenin $(\mathrm{P}<0.001)$, whereas no association was observed between STIM1 and MMP-9 expression ( $\mathrm{P}>0.05)$. In conclusion, the results of the present study suggested that STIM1 may be a valuable prognostic marker in GC patients, and that STIM1 may increase GC motility and invasiveness by promoting epithelial-mesenchymal transition.
\end{abstract}

Correspondence to: Dr Yong Li, Department of General Surgery, The Fourth Affiliated Hospital, Hebei Medical University, 12 Jian-Kang Road, Shijiazhuang, Hebei 050011, P.R. China E-mail: 1i_yong_hbth@126.com

Key words: $\beta$-catenin, E-cadherin, epithelial-mesenchymal transition, gastric cancer, overall survival, stromal interaction molecule 1, matrix metalloproteinase- 9

\section{Introduction}

Gastric cancer (GC) is the fourth most common cancer and the second leading cause of cancer-associated mortality worldwide (1). As 5-year survival rates of GC remain $<30 \%(2,3)$, further understanding of $\mathrm{GC}$ is urgently required.

Epithelial-mesenchymal transition (EMT) is an essential early step in tumor metastasis (4). During EMT, tumor cells lose their epithelial characteristics and obtain mesenchymal traits (5-8). It has been demonstrated that EMT is correlated with poor tumor staging, an increased risk of cancer recurrence and decreased survival in various cancer types, including breast $(9,10)$, colorectal (11), bladder $(12,13)$, lung (14) and GC (15).

Stromal interaction molecule 1 (STIM1) is responsible for the activation of store-operated $\mathrm{Ca}^{2+}$ entry (16). Previous studies have reported that STIM1 is important in the growth and migration of cancer cells, and for angiogenesis and progression of cancer (17-20). Furthermore, STIM1 is a key molecule in the process of EMT in various cancer types. Ectopic STIM1 expression induced EMT in colorectal cancer cells (20), and STIM1 silencing reversed EMT initiated by downregulation of the POU class 5 homeobox 1 transcription factor in breast cancer cells (19). Casas-Rua et al (21) demonstrated that STIM1 overexpression increased migration and EMT in endometrial adenocarcinoma cells. However, the role of STIM1 in GC progression and metastasis and its association with EMT remains to be elucidated.

In the present study, immunohistochemistry was performed to detect STIM1, E-cadherin, $\beta$-catenin and matrix metalloproteinase-9 (MMP-9) in 170 GC and 35 adjacent healthy gastric tissue samples. STIM1 was overexpressed in GC samples and associated with poor survival of GC patients. STIM1 expression was significantly associated with abnormal cytoplasmic and nuclear expression of E-cadherin and $\beta$-catenin in GC cells, which suggested that STIM1 may promote EMT in GC.

\section{Materials and methods}

Patients and tissue samples. GC and adjacent healthy tissue samples were obtained from $170 \mathrm{GC}$ patients with histologically confirmed gastric adenocarcinoma between June 
2009 and October 2011 at The Fourth Affiliated Hospital of Hebei Medical University (Shijiazhuang, China). All patients underwent surgical resection of the stomach with lymph node dissection, with no chemotherapy or radiotherapy preoperation; no other cancers were diagnosed simultaneously. The present study was approved by the Ethics Committees of The Fourth Affiliated Hospital of Hebei Medical University, and written consent was obtained from all patients. Follow-up data were primarily obtained by telephone and out-patient review. Patients with inadequate follow-up were excluded from the study.

Of the $170 \mathrm{GC}$ patients enrolled in the present study, 127 were male $(74.7 \%)$ and 43 were female $(25.3 \%)$, with an average age of 57.85 years (range, 33-81 years). In total, $57(33.5 \%)$ cases were stage I and II tumors at diagnosis, and $113(66.5 \%)$ were stage III and IV. Tumors were evaluated according to the tumor-node-metastasis (TNM) staging system for carcinoma of the stomach (22). A total of 106 patients (62.4\%) had regional lymph node metastasis (LNM), whereas 64 (37.6\%) had no regional LNM. Tumors were located in the cardia of $54.1 \%$ of patients, and in the antrum of $39.4 \%$. Tumors ranged from 2 to $12 \mathrm{~cm}$ in size, with a mean size of $7.12 \mathrm{~cm}$. A total of 79 tumors (46.5\%) were poorly differentiated and $91(53.5 \%)$ were moderately or well differentiated, according to the criteria proposed by the World Health Organization Classification of Tumors (3rd Edition) (23). The clinicopathological characteristics of patients are presented in Table I.

$170 \mathrm{GC}$ tumor tissues were analyzed in the present study, with 35 adjacent healthy gastric mucosa tissues as negative controls. All tissue samples were fixed in $10 \%$ neutral formalin, embedded in paraffin blocks, cut into $4-\mu \mathrm{m}$ thick serial sections, and placed on glass slides for immunohistochemical staining.

Immunohistochemical staining of STIM1, E-cadherin, $\beta$-catenin and MMP-9. Immunohistochemistry was performed using rabbit anti-human STIM1 (ab108994; 1:100; Abcam, Cambridge, UK), mouse anti-human E-cadherin (ab1416; 1:100; Abcam), rabbit anti-human anti- $\beta$-catenin (ab32572; 1:500; Abcam) and rabbit anti-human MMP-9 (ab73734; 1:200; Abcam) primary antibodies. Sections were deparaffinized, rehydrated, rinsed in phosphate-buffered saline (PBS; $\mathrm{pH}$ 7.4) and autoclaved in EDTA buffer ( $\mathrm{pH}$ 8.0) for antigen retrieval. Following cooling to room temperature for $20 \mathrm{~min}$, sections were rinsed in PBS and incubated in $3 \% \mathrm{H}_{2} \mathrm{O}_{2}$ for 15 min to block endogenous peroxidase activity. Sections were again rinsed with PBS and incubated with normal goat serum (Beijing Zhongshan Jinqiao Biological Technology Co., Ltd., Beijing, China) at $37^{\circ} \mathrm{C}$ for 15 min to block nonspecific antibody binding. Following incubation with primary antibodies at $37^{\circ} \mathrm{C}$ for $2 \mathrm{~h}$, sections were rinsed in PBS, incubated with a biotinylated secondary antibody (biotinylated goat anti-mouse/rabbit IgG; SP-9000; Beijing Zhongshan Jinqiao Biological Technology Co., Ltd.) at room temperature for 15 min and rinsed with PBS. Following incubation with streptavidin-horseradish peroxidase (Beijing Zhongshan Jinqiao Biological Technology Co., Ltd.) and further rinsing with PBS, proteins were visualized using 3,3'-diaminobenzidine (Beijing Zhongshan Jinqiao Biological Technology Co., Ltd.) and sections were counterstained with hematoxylin. Finally, sections were dehydrated, cleared, covered with coverslips and sealed with neutral gum.

All slides were assessed by two pathologists who were blinded to the patient details. The intensity of STIM1 staining was graded on a $0-3$ scale: 0 , no staining; 1 , weak immunoreactivity; 2 , moderate immunoreactivity; 3 , strong immunoreactivity. The percentage of immunoreactivity was scored on a $0-3$ scale: 0 , no positive cells; $1,0-25 \%$ positive cells; $2,26-50 \%$ positive cells; $3,>50 \%$ positive cells (24). E-cadherin, $\beta$-catenin and MMP-9 staining were classified as abnormal according to the degree of cytoplasmic and nuclear staining and the proportion of positive cells. Abnormal staining intensity was graded on a 0-3 scale: 0 , no staining; 1 , weak immunoreactivity; 2 , moderate immunoreactivity; 3 , strong immunoreactivity. The percentage of abnormal immunoreactivity was scored on a $0-4$ scale: $0,0-20 \%$ positive cells; $1,21-40 \%$ positive cells; $2,41-60 \%$ positive cells; $3,61-80 \%$ positive cells; $4,>80 \%$ positive cells (25). The staining intensity score was multiplied by the percentage immunoreactivity score to obtain a composite score. The composite score was considered the overall expression level: 0-4, negative; 5-6, positive; 6-12, strongly positive.

Statistical analysis. All data were processed with SPSS software version 19.0 (IBM SPSS, Armonk, NY, USA). P $<0.05$ was considered to indicate a statistically significant difference. The chi-square test was used to analyze the association between STIM1 expression and patient characteristics. A binary logistical regression model was applied to identify factors associated with STIM1 positive expression. Cohen's kappa statistic was used to determine the association between STIM1 expression and abnormal E-cadherin and $\beta$-catenin expression. The Kaplan-Meier method was used to calculate patient survival rate, and the Cox proportional hazards models were employed to identify independent factors associated with patient survival. In this model, X1, Age; X2, Sex; X3, Tumor location; X4, Tumor differentiation; X5, Tumor size; X6, Lymphatic metastasis; $\mathrm{X} 7$, Tumor-node-metastasis; and $\mathrm{X} 8$, STIM1 expression were used as independent variables; and Y, Survival as a dependent variable.

\section{Results}

STIM1 expression and its association with clinicopathological characteristics of GC patients. STIM1 expression in GC tissues was predominantly cytoplasmic (Fig. 1A). The STIM1 positive expression rate in GC tissues was $43.5 \%$ (74/170), which was significantly greater compared with adjacent healthy tissues $\left(8.60 \% ; 3 / 35 ; \chi^{2}=15.12 ; \mathrm{P}<0.001\right.$; Table II; Fig. 1B). The STIM1 expression rate in GC patients with LNM was significantly greater compared with patients without LNM $(\mathrm{P}<0.001)$. STIM1 expression in stage I-II GC tissues was $33.5 \%$ (17/57), which was significantly reduced compared with stage III-IV tumors (66.5\%; 57/113; P=0.01; Table I). However, STIM1 expression in GC tissues did not correlate with sex, age, the degree of histologic differentiation, location of the tumor or tumor size $(\mathrm{P}>0.05)$. Cox risk regression analysis indicated that lymphatic metastasis was the only independent risk factor for STIM1 expression in GC patients (Table III). 


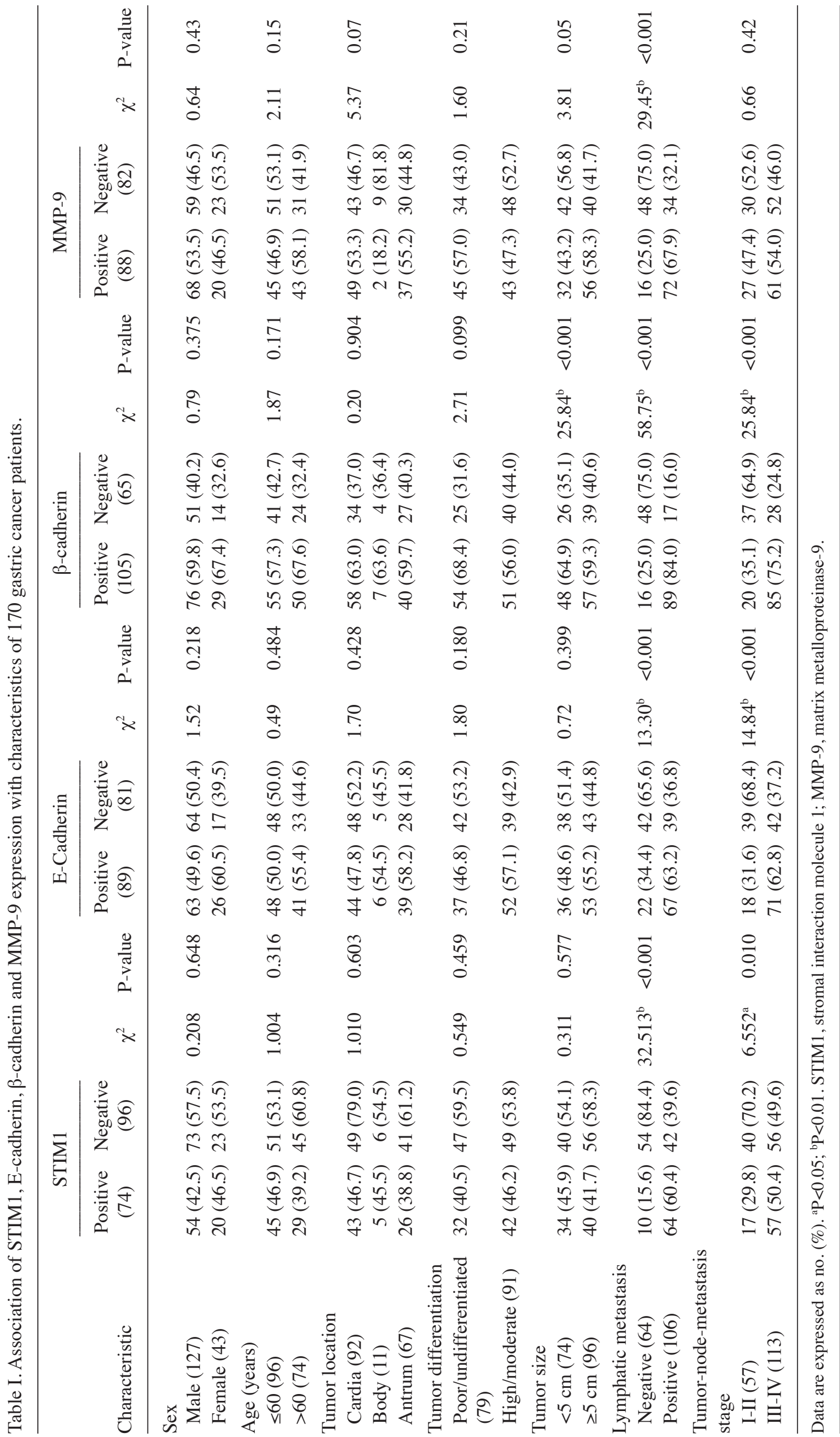


E-cadherin, $\beta$-catenin and MMP-9 expression, and their association with clinicopathological features of GC patients. In the present study, E-cadherin (Fig. $1 \mathrm{C}$ and D) and $\beta$-catenin (Fig. 1E and F) were abnormally expressed in the cytoplasm or nucleus of GC cells. The positive expressions of E-cadherin and $\beta$-catenin were observed in $61.8 \%(105 / 170)$ and $52.4 \%$ (89/170), respectively, of GC tissue samples, and in $11.4 \%(4 / 35)$ and $20.0 \%(7 / 35)$, respectively, of adjacent healthy gastric tissues. Differences in the rates of abnormal E-cadherin and $\beta$-catenin expression between GC tissues and adjacent healthy gastric tissues were significant $(\mathrm{P}<0.001$; Table II). MMP-9 expression in GC tissues was additionally predominantly cytoplasmic (Fig. 1G). Greater expression of MMP-9 was observed in GC tissues compared with adjacent healthy gastric tissues $(\mathrm{P}<0.05$; Table II; Fig. $1 \mathrm{H})$. In addition, expression of E-cadherin was positively associated with LNM and a more advanced clinical stage $(\mathrm{P}<0.001$; Table I). $\beta$-catenin expression correlated significantly with tumor size, LNM and the clinical stage of GC tissues $(\mathrm{P}<0.001$; Table I); however, there was no correlation between $\beta$-catenin expression and other clinicopathological parameters ( $\mathrm{P}>0.05$; Table I). Expression of MMP-9 was positively associated with LNM $(\mathrm{P}<0.001$; Table I); however, there was no correlation between MMP-9 expression and other clinicopathological parameters $(\mathrm{P}>0.05$; Table I).

Associations between STIM1, E-cadherin, $\beta$-catenin and MMP-9 expression in GC tissues. Potential associations between STIM1, E-cadherin and $\beta$-catenin expression patterns in GC were evaluated. Of STIM1-positive tumors, 78.4\% (58/74) were E-cadherin positive and 90.5\% (67/74) were positive for $\beta$-catenin. Chi-square tests revealed that STIM1 expression correlated significantly with abnormal E-cadherin expression $\left(\chi^{2}=34.555 ; \mathrm{P}<0.001 ; \kappa=0.447\right)$ and with abnormal $\beta$-catenin expression $\left(\chi^{2}=45.947 ; \mathrm{P}<0.001 ; \kappa=0.486\right.$; Table IV), whereas no correlation was observed between STIM1 and MMP-9 $\left(\chi^{2}=1.420 ; \mathrm{P}=0.233 ; \kappa=-0.616\right.$; Table IV $)$. Furthermore, $79.8 \%(71 / 89)$ of E-cadherin-positive tumors were additionally positive for $\beta$-catenin, and this association was statistically significant $(\mathrm{P}<0.05$; Table $\mathrm{V})$.

Association between STIM1 expression and survival. Using Kaplan-Meier analysis, it was demonstrated that the overall survival rate was significantly reduced in patients with STIM1-expressing GC tumors compared with patients with GC tumors without STIM1 expression ( $\mathrm{P}=0.043$; Fig. 2). Factors that significantly correlated with patient survival rate, including STIM1 expression, LNM and a high TNM stage, were identified by univariate analysis (Table VI). Cox risk regression analysis indicated that STIM1 expression and LNM were independent prognostic factors for GC patients (Table VII).

\section{Discussion}

Various studies have demonstrated that STIM1 protein is involved in adhesion, invasion, metastasis and proliferation of cancer cells $(17,18,26-29)$. STIM1 expression has been reported to correlate with lymphatic invasion in colon adenocarcinomas (30). Ectopic STIM1 overexpression in colorectal 
Table III. Multivariate analysis of factors associated with stromal interaction molecule 1 expression in gastric carcinoma.

\begin{tabular}{lcccccccr}
\hline & & & & & & & & \multicolumn{2}{c}{$\begin{array}{c}95.0 \% \text { CI } \\
\text { for Exp (B) }\end{array}$} \\
\cline { 3 - 9 } Parameter & B & SE & Wald & df & Sig. & Exp (B) & Lower & Upper \\
\hline Sex & 0.690 & 0.429 & 2.584 & 1 & 0.108 & 1.994 & 0.860 & 4.626 \\
Age & -0.403 & 0.359 & 1.256 & 1 & 0.262 & 0.669 & 0.331 & 1.352 \\
Tumor location & -0.028 & 0.190 & 0.023 & 1 & 0.881 & 0.972 & 0.670 & 1.410 \\
Tumor differentiation & 0.162 & 0.358 & 0.205 & 1 & 0.651 & 1.176 & 0.583 & 2.371 \\
Tumor size & -0.126 & 0.361 & 0.121 & 1 & 0.728 & 0.882 & 0.434 & 1.790 \\
Lymphatic metastasis & 2.171 & 0.446 & 23.734 & 1 & 0.000 & 8.767 & 3.660 & 20.998 \\
Tumor-node-metastasis & 0.238 & 0.411 & 0.336 & 1 & 0.562 & 1.269 & 0.567 & 2.840 \\
Constant & -2.666 & 1.154 & 5.335 & 1 & 0.021 & 0.070 & & \\
\hline
\end{tabular}

CI, confidence interval. B, regression coefficient; SE, standard error; Wald, the statistic value of the regression; df, degree of freedom; Sig, significance; $\operatorname{Exp}(\mathrm{B})$, odds ratio.

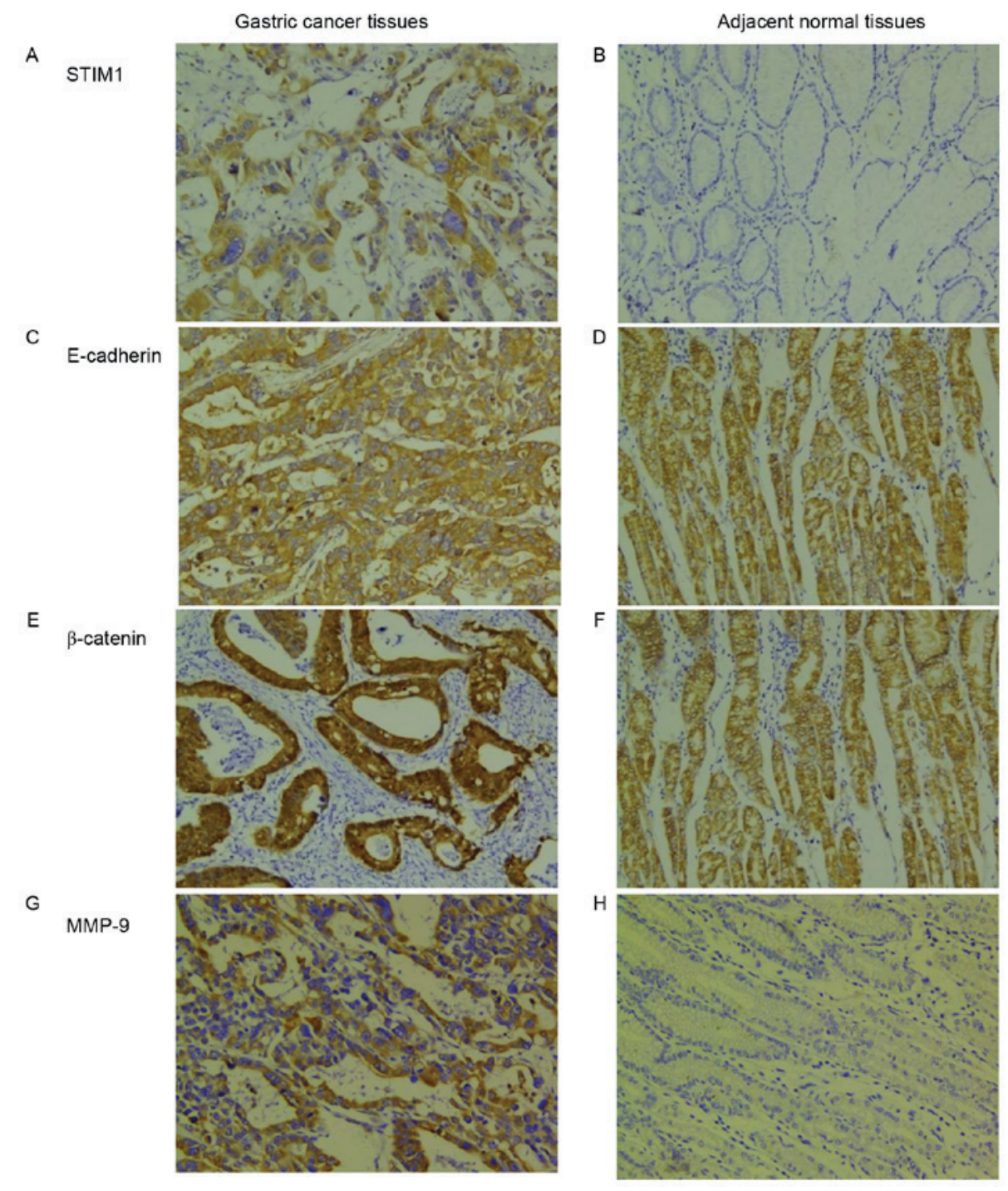

Figure 1. Immunohistochemical staining of STIM1, E-cadherin, $\beta$-catenin and MMP-9 in GC and adjacent healthy gastric tissues. STIM1 expression was detected in (A) GC tissues, but not in (B) adjacent healthy gastric tissues. E-cadherin expression was abnormal in (C) GC tissues and normal in (D) adjacent healthy gastric tissues. $\beta$-catenin expression was abnormal in (E) GC tissues and normal in (F) adjacent healthy gastric tissues. MMP-9 expression was detected in (G) GC tissues, but not in (H) adjacent healthy gastric tissues. Original magnification, x200. STIM1, stromal interaction molecule 1; MMP-9, matrix metalloproteinase-9; GC, gastric cancer. 
Table V. E-cadherin and $\beta$-catenin expression in 170 gastric cancer samples.

\begin{tabular}{lcccc}
\hline & \multicolumn{2}{c}{$\beta$-cadherin } & & \\
\cline { 2 - 3 } Parameter & Positive & Negative & $\chi^{2}$ & P-value \\
\hline E-cadherin (+) & $71(79.8)$ & $18(20.2)$ & $4.92^{\mathrm{a}}$ & 0.03 \\
E-cadherin (-) & $34(42.0)$ & $47(58.0)$ & & \\
\hline
\end{tabular}

Data are expressed as no. (\%). ${ }^{\mathrm{a}} \mathrm{P}<0.05$.

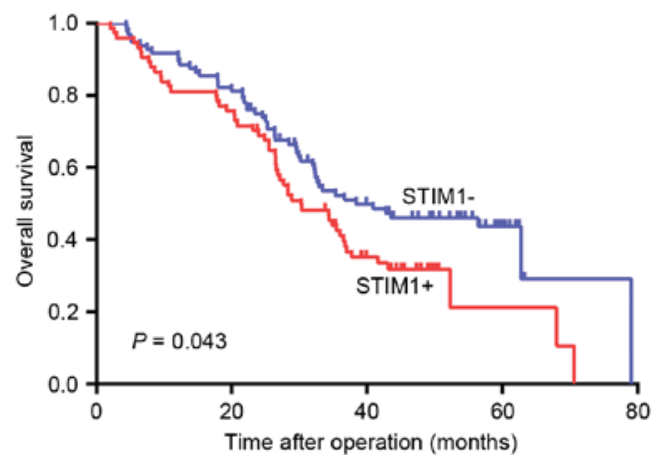

Figure 2. Overall survival of STIM1-positive and -negative patients The overall survival rate was significantly reduced in patients with STIM1-expressing GC tumors compared with patients with GC tumors without STIM1 expression ( $\mathrm{P}=0.043)$. STIM1, stromal interaction molecule 1; GC, gastric cancer.

cancer was revealed to significantly associate with tumor size, depth of invasion and LNM status, and to promote colorectal cancer cell motility (24). It has been reported that STIM1 is upregulated during hepatocarcinoma growth (31), and STIM1 has been suggested to be critical for breast cancer cell migration and metastasis (18). However, certain studies have demonstrated that STIM1 protein serves an opposing role in various cancers. For example, in vitro overexpression of STIM1 in G401 rhabdomyosarcoma cells resulted in morphological alterations and, ultimately, cell death $(32,33)$. Suyama et al (34) revealed that STIM1 has an antimetastatic function. Weidinger et al (35) reported that patients with loss-of-function mutations in the STIM1 gene were immunodeficient and prone to developing virus-associated tumors. The present study demonstrated that STIM1 was highly expressed in GC compared with adjacent healthy tissues, and that STIM1 expression was associated with LNM, TNM stage and poor overall survival rate. Furthermore, LNM was the only independent risk factor for STIM1 expression in GC patients. The results of the present study indicated that STIM1 may serve an important role in the initiation and development of GC, and may contribute to the diagnosis and treatment of GC as a prognostic marker. These results therefore provide novel information on the function of STIM1 in GC progression.

The molecular mechanisms underlying the effect of STIM1 on the process of EMT in GC remain to be fully elucidated. A previous study by Hu et al (19) suggested that STIM1 may be involved in EMT, which is a critical step in immune evasion 
Table VI. Univariate analysis of prognostic factors for gastric carcinoma.

\begin{tabular}{|c|c|c|c|c|}
\hline Characteristic & Succumbed to disease (104) & Survival rate at 80 months (66) & $\chi^{2}$ & P-value \\
\hline \multicolumn{5}{|l|}{ Sex } \\
\hline Male & $79(76.0)$ & $48(72.7)$ & 0.224 & 0.636 \\
\hline Female & $25(24.0)$ & $18(27.3)$ & & \\
\hline \multicolumn{5}{|l|}{ Age (years) } \\
\hline$\leq 60$ & $58(55.8)$ & $38(57.6)$ & 0.054 & 0.817 \\
\hline$>60$ & $46(44.2)$ & $28(42.4)$ & & \\
\hline \multicolumn{5}{|l|}{ Tumor location } \\
\hline Cardia & $58(55.8)$ & $34(51.5)$ & 0.400 & 0.819 \\
\hline Body & $6(5.8)$ & $5(5.8)$ & & \\
\hline Antrum & $40(38.5)$ & $27(40.9)$ & & \\
\hline \multicolumn{5}{|l|}{ Tumor differentiation } \\
\hline Poor/undifferentiated & $49(47.1)$ & $30(45.5)$ & 0.045 & 0.832 \\
\hline High/moderate & $55(52.9)$ & $36(54.5)$ & & \\
\hline \multicolumn{5}{|l|}{ Tumor size } \\
\hline$<5 \mathrm{~cm}$ & $47(45.2)$ & $27(40.9)$ & 0.301 & 0.583 \\
\hline$\geq 5 \mathrm{~cm}$ & $57(54.8)$ & $39(59.1)$ & & \\
\hline \multicolumn{5}{|l|}{ Lymphatic metastasis } \\
\hline Negative & $15(14.4)$ & $49(74.2)$ & $61.549^{\mathrm{b}}$ & $<0.001$ \\
\hline Positive & $89(85.6)$ & $17(25.8)$ & & \\
\hline \multicolumn{5}{|c|}{ Tumor-node-metastasis stage } \\
\hline I-II & $14(13.5)$ & $43(65.2)$ & $48.404^{b}$ & $<0.001$ \\
\hline III-IV & $90(86.5)$ & $23(34.8)$ & & \\
\hline \multicolumn{5}{|l|}{ STIM1 expression } \\
\hline Positive & $52(50.0)$ & $22(33.3)$ & $4.563^{\mathrm{a}}$ & 0.033 \\
\hline Negative & $52(50.0)$ & $44(66.7)$ & & \\
\hline
\end{tabular}

Data are expressed as no. (\%). ${ }^{\mathrm{a}}<0.05$; ${ }^{\mathrm{P}}<0.01$. STIM1, stromal interaction molecule 1.

Table VII. Multivariate analysis of prognostic factors for gastric carcinoma.

$95.0 \%$ CI for HR

\begin{tabular}{lcccccrrr} 
Parameter & B & SE & Wald & df & Sig. & HR & Lower & Upper \\
\hline Age & 0.292 & 0.209 & 1.950 & 1 & 0.163 & 1.339 & 0.889 & 2.018 \\
Sex & -0.221 & 0.259 & 0.728 & 1 & 0.394 & 0.802 & 0.483 & 1.331 \\
Tumor location & -0.030 & 0.107 & 0.079 & 1 & 0.778 & 0.970 & 0.786 & 1.197 \\
Tumor differentiation & 0.047 & 0.208 & 0.050 & 1 & 0.823 & 1.048 & 0.696 & 1.577 \\
Tumor size & 0.017 & 0.211 & 0.006 & 1 & 0.936 & 1.017 & 0.672 & 1.539 \\
Lymphatic metastasis & 1.699 & 0.328 & 26.797 & 1 & 0.000 & 5.468 & 2.874 & 10.403 \\
Tumor-node-metastasis & 1.307 & 0.311 & 17.651 & 1 & 0.000 & 3.695 & 2.008 & 6.799 \\
STIM1 expression & 0.095 & 0.286 & 0.111 & 1 & 0.039 & 1.100 & 1.020 & 1.328
\end{tabular}

CI, confidence interval; STIM1, stromal interaction molecule 1. B, regression coefficient. SE, standard error. Wald, the statistic value of the regression. df, degree of freedom. Sig, significance. HR, hazard ratio.

and metastasis of tumor cells. In addition, STIM1 overexpression has been reported to induce EMT in colorectal cancer cells, whereas STIM1 silencing had the opposite effect (20).
Casas-Rua et al (21) demonstrated that STIM1 phosphorylation at extracellular signal-regulated kinase 1/2 target sites mediates EMT triggered by epidermal growth factor in Ishikawa 
cells. However, the role of STIM1 in cancer cell progression and metastasis and its association with EMT in GC remain to be investigated. In the present study, the association between the expression of STIM1, E-cadherin, $\beta$-catenin and MMP-9 proteins in GC tissues was analyzed by immunohistochemical staining. The results of the present study revealed that STIM1 overexpression in GC tissues correlated significantly with abnormal E-cadherin and $\beta$-catenin expression in the cytoplasm and nucleus, whereas no association was observed between STIM1 and MMP-9 expression. Therefore, STIM1 may increase GC motility and invasiveness by promoting EMT via E-cadherin and $\beta$-cadherin; however, MMP-9 does not appear to be involved in this process.

In conclusion, the results of the present study demonstrated that STIM1 is significantly upregulated in GC and that STIM1 overexpression is associated with a poor prognosis in GC patients with LNM and an advanced TNM stage. Therefore, STIM1 may be a useful prognostic marker for GC.

\section{References}

1. Ferlay J, Soerjomataram I, Dikshit R, Eser S, Mathers C, Rebelo M, Parkin DM, Forman D and Bray F: Cancer incidence and mortality worldwide: Sources, methods and major patterns in GLOBOCAN 2012. Int J Cancer 136: E359-E386, 2015.

2. Suzuki R, Yamamoto E, Nojima M, Maruyama R, Yamano HO, Yoshikawa K, Kimura T, Harada T, Ashida M, Niinuma T, et al: Aberrant methylation of microRNA-34b/c is a predictive marker of metachronous gastric cancer risk. J Gastroenterol 49: 1135-1144, 2014

3. Bria E, De Manzoni G, Beghelli S, Tomezzoli A, Barbi S, Di Gregorio C, Scardoni M, Amato E, Frizziero M, Sperduti I, et al: A clinical-biological risk stratification model for resected gastric cancer: Prognostic impact of Her2, Fhit, and APC expression status. Ann Oncol 24: 693-701, 2013.

4. Kraljevic Pavelic S, Sedic M, Bosnjak H, Spaventi S and Pavelic K: Metastasis: New perspectives on an old problem. Mol Cancer 10: 22, 2011.

5. Thiery JP, Acloque H, Huang RY and Nieto MA: Epithelial-mesenchymal transitions in development and disease. Cell 139: 871-890, 2009.

6. Thiery JP and Sleeman JP: Complex networks orchestrate epithelial-mesenchymal transitions. Nat Rev Mol Cell Biol 7: 131-142, 2006.

7. Savagner P, Boyer B, Valles AM, Jouanneau J and Thiery JP: Modulations of the epithelial phenotype during embryogenesis and cancer progression. Cancer Treat Res 71: 229-249, 1994.

8. Tam WL and Weinberg RA: The epigenetics of epithelial-mesenchymal plasticity in cancer. Nat Med 19: 1438-1449, 2013.

9. Liu J, Chen X, Ward T, Pegram M and Shen K: Combined niclosamide with cisplatin inhibits epithelial-mesenchymal transition and tumor growth in cisplatin-resistant triple-negative breast cancer. Tumour Biol 37: 9825-9835, 2016.

10. Luo M, Hou L, Li J, Shao S, Huang S, Meng D, Liu L, Feng L, Xia P, Qin T and Zhao X: VEGF/NRP-1axis promotes progression of breast cancer via enhancement of epithelial-mesenchymal transition and activation of NF- $\mathrm{KB}$ and $\beta$-catenin. Cancer Lett 373: 1-11, 2016.

11. Ma J, Zhao J, Lu J, Wang P, Feng H, Zong Y, Ou B, Zheng M and Lu A: Cadherin-12 enhances proliferation in colorectal cancer cells and increases progression by promoting EMT. Tumour Biol 37: 9077-9088, 2016.

12. Bruyere F, Namdarian B, Corcoran NM, Pedersen J, Ockrim J, Voelzke BB, Mete U, Costello AJ and Hovens CM: Snail expression is an independent predictor of tumor recurrence in superficial bladder cancers. Urol Oncol 28: 591-596, 2010.

13. Chiu KY, Wu CC, Chia CH, Hsu SL and Tzeng YM: Inhibition of growth, migration and invasion of human bladder cancer cells by antrocin, a sesquiterpene lactone isolated from Antrodia cinnamomea, and its molecular mechanisms. Cancer Lett 373 174-184, 2016.
14. Lee SO, Yang X, Duan S, Tsai Y, Strojny LR, Keng P and Chen Y: IL-6 promotes growth and epithelial-mesenchymal transition of CD133+ cells of non-small cell lung cancer. Oncotarget 7: 6626-6638, 2016.

15. Huang L, Wu RL and Xu AM: Epithelial-mesenchymal transition in gastric cancer. Am J Transl Res 7: 2141-2158, 2015.

16. Parekh AB: Store-operated CRAC channels: Function in health and disease. Nat Rev Drug Discov 9: 399-410, 2010.

17. Chen YF, Chiu WT, Chen YT, Lin PY, Huang HJ, Chou CY, Chang HC, Tang MJ and Shen MR: Calcium store sensor stromal-interaction molecule 1-dependent signaling plays an important role in cervical cancer growth, migration, and angiogenesis. Proc Natl Acad Sci USA 108: 15225-15230, 2011.

18. Yang S, Zhang JJ and Huang XY: Orail and STIM1 are critical for breast tumor cell migration and metastasis. Cancer Cell 15: 124-134, 2009.

19. Hu J, Qin K, Zhang Y, Gong J, Li N, Lv D, Xiang R and Tan X: Downregulation of transcription factor Oct4 induces an epithelial-to-mesenchymal transition via enhancement of $\mathrm{Ca} 2+$ influx in breast cancer cells. Biochem Biophys Res Commun 411: 786-791, 2011.

20. Zhang Z, Liu X, Feng B, Liu N, Wu Q, Han Y, Nie Y, Wu K, Shi Y and Fan D: STIM1, a direct target of microRNA-185, promotes tumor metastasis and is associated with poor prognosis in colorectal cancer. Oncogene 34: 4808-4820, 2015.

21. Casas-Rua V, Tomas-Martin P, Lopez-Guerrero AM, Alvarez IS, Pozo-Guisado E and Martin-Romero FJ: STIM1 phosphorylation triggered by epidermal growth factor mediates cell migration. Biochim Biophys Acta 1853: 233-243, 2015.

22. Edge S, Byrd DR, Compton CC, Fritz AG, Greene FL and Trotti A: AJCC Cancer Staging Handbook. Springer-Verlag New York, Chicago, 2010.

23. World Health Organization Classification of Tumours: Pathology and genetics of tumours of the digestive system. IARC Press, Lyon, 2000.

24. Wang JY, Sun J, Huang MY, Wang YS, Hou MF, Sun Y, He H, Krishna N, Chiu SJ, Lin S et al: STIM1 overexpression promotes colorectal cancer progression, cell motility and COX-2 expression. Oncogene 34: 4358-4367, 2015

25. Chaw SY, Majeed AA, Dalley AJ, Chan A, Stein S and Farah CS: Epithelial to mesenchymal transition (EMT) biomarkers-E-cadherin, beta-catenin, APC and Vimentin-in oral squamous cell carcinogenesis and transformation. Oral Oncol 48: 997-1006, 2012.

26. Ouadid-Ahidouch H, Dhennin-Duthille I, Gautier M, Sevestre H and Ahidouch A: TRP channels: Diagnostic markers and therapeutic targets for breast cancer? Trends Mol Med 19: 117-124, 2013.

27. Prevarskaya N, Skryma R and Shuba Y: Calcium in tumour metastasis: New roles for known actors. Nat Rev Cancer 11: 609-618, 2011.

28. Bergmeier W, Weidinger C, Zee I and Feske S: Emerging roles of store-operated $\mathrm{Ca}^{2+}$ entry through STIM and ORAI proteins in immunity, hemostasis and cancer. Channels (Austin) 7: 379-391, 2013.

29. Liu H, Hughes JD, Rollins S, Chen B and Perkins E: Calcium entry via ORAI1 regulates glioblastoma cell proliferation and apoptosis. Exp Mol Pathol 91: 753-760, 2011.

30. Wong HS and Chang WC: Correlation of clinical features and genetic profiles of stromal interaction molecule 1 (STIM1) in colorectal cancers. Oncotarget 6: 42169-42182, 2015.

31. Li Y, Guo B, Xie Q, Ye D, Zhang D, Zhu Y, Chen H and Zhu B: STIM1 Mediates Hypoxia-Driven Hepatocarcinogenesis via Interaction with HIF-1. Cell Rep 12: 388-395, 2015.

32. Sabbioni S, Barbanti-Brodano G, Croce CM and Negrini M: GOK: A gene at 11p15 involved in rhabdomyosarcoma and rhabdoid tumor development. Cancer Res 57: 4493-4497, 1997.

33. Sabbioni S, Veronese A, Trubia M, Taramelli R, Barbanti-Brodano G, Croce CM and Negrini M: Exon structure and promoter identification of STIM1 (alias GOK), a human gene causing growth arrest of the human tumor cell lines G401 and RD. Cytogenet Cell Genet 86: 214-218, 1999.

34. Suyama E, Wadhwa R, Kaur K, Miyagishi M, Kaul SC, Kawasaki $\mathrm{H}$ and Taira K: Identification of metastasis-related genes in a mouse model using a library of randomized ribozymes. J Biol Chem 279: 38083-38086, 2004.

35. Weidinger C, Shaw PJ and Feske S: STIM1 and STIM2-mediated $\mathrm{Ca}(2+)$ influx regulates antitumour immunity by $\mathrm{CD} 8(+) \mathrm{T}$ cells EMBO Mol Med 5: 1311-1321, 2013. 